\title{
Energetic ions in the Venusian system: Insights from the first Solar Orbiter flyby
}

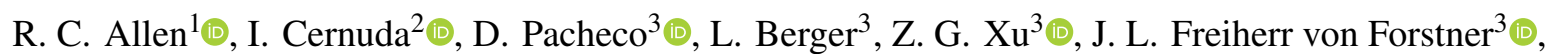 \\ J. Rodríguez-Pacheco ${ }^{2} \oplus$, R. F. Wimmer-Schweingruber ${ }^{3} \oplus$, G. C. Ho ${ }^{1}$, G. M. Mason ${ }^{1} \oplus$, S. K. Vines ${ }^{1} \oplus$,

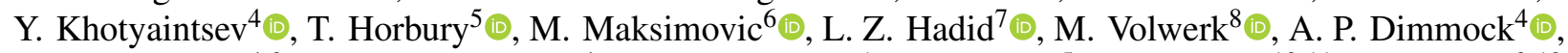

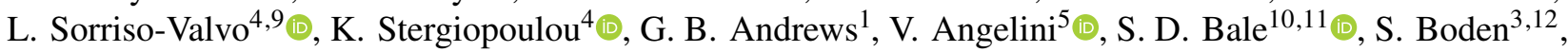

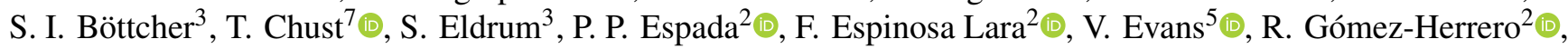 \\ J. R. Hayes ${ }^{1}$, A. M. Hellín² ${ }^{\circledR}$, A. Kollhoff ${ }^{3}$, V. Krasnoselskikh ${ }^{13}$, M. Kretzschmarr'13,14, P. Kühl ${ }^{3}$, \\ S. R. Kulkarni ${ }^{3,15}$, W. J. Lees ${ }^{1}$, E. Lorfèvre ${ }^{16}$, C. Martin ${ }^{3,17}$, H. O’Brien ${ }^{5}$, D. Plettemeier ${ }^{18}$, O. R. Polo ${ }^{2} \odot$, \\ M. Prieto ${ }^{2} \odot$, A. Ravanbakhsh ${ }^{3,19}$, S. Sánchez-Prieto ${ }^{2}$, C. E. Schlemm ${ }^{1}$, H. Seifert ${ }^{1}$, J. Souček ${ }^{20} \odot$, M. Steller ${ }^{8}$,

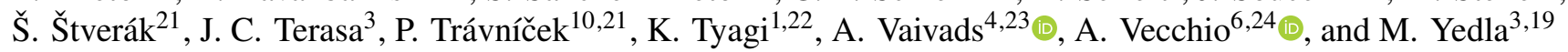 \\ (Affiliations can be found after the references)
}

Received 13 March 2021 / Accepted 30 April 2021

\begin{abstract}
The Solar Orbiter flyby of Venus on 27 December 2020 allowed for an opportunity to measure the suprathermal to energetic ions in the Venusian system over a large range of radial distances to better understand the acceleration processes within the system and provide a characterization of galactic cosmic rays near the planet. Bursty suprathermal ion enhancements (up to $\sim 10 \mathrm{keV}$ ) were observed as far as $\sim 50 R_{V}$ downtail. These enhancements are likely related to a combination of acceleration mechanisms in regions of strong turbulence, current sheet crossings, and boundary layer crossings, with a possible instance of ion heating due to ion cyclotron waves within the Venusian tail. Upstream of the planet, suprathermal ions are observed that might be related to pick-up acceleration of photoionized exospheric populations as far as $5 R_{V}$ upstream in the solar wind as has been observed before by missions such as Pioneer Venus Orbiter and Venus Express. Near the closest approach of Solar Orbiter, the Galactic cosmic ray (GCR) count rate was observed to decrease by approximately 5 percent, which is consistent with the amount of sky obscured by the planet, suggesting a negligible abundance of GCR albedo particles at over $2 R_{V}$. Along with modulation of the GCR population very close to Venus, the Solar Orbiter observations show that the Venusian system, even far from the planet, can be an effective accelerator of ions up to $\sim 30 \mathrm{keV}$. This paper is part of a series of the first papers from the Solar Orbiter Venus flyby.
\end{abstract}

Key words. acceleration of particles - planets and satellites: terrestrial planets - planet-star interactions - planetary systems - turbulence - waves

\section{Introduction}

The interactions between planets and stellar winds from their host star are a fundamental aspect in space physics. Venus and Mars, as opposed to the other planets in our Solar System, do not have intrinsic magnetic fields. Unlike Mars, Venus does have a substantial ionosphere, formed by the photoionization of its thick atmosphere, that supports current systems generated by the variable solar wind. This induced magnetic field, in turn, acts to establish the near-Venus plasma environment (see reviews by Luhmann 1986; Phillips \& McComas 1991; Bertucci et al. 2011; Dubinin et al. 2011; Futaana et al. 2017).

The interaction between the solar wind and Venus establishes several distinct regions. The deflection of the supersonic and super-Alfvénic solar wind from the induced magnetosphere creates a bow shock that is about a tenth of the size of Earth's bow shock (Slavin et al. 1979) and is far weaker than at Earth (Russell et al. 1979; Lu et al. 2013). The shocked solar wind then forms the magnetosheath, comprised of both solar windoriginating ions as well as picked-up ions from the Venusian atmosphere (Gröller et al. 2010). The magnetosheath is characterized by hot dense plasma (Phillips \& McComas 1991) and a highly turbulent fluctuating magnetic field consisting of mirror-mode waves (Volwerk et al. 2008) with different spectral and scaling properties (Vörös et al. 2008).

As the interplanetary magnetic field (IMF) is carried towards the planet by the magnetosheath plasma, a pile-up region is created along the dayside of Venus forming a magnetic barrier (Zhang et al. 2008; Bertucci et al. 2003) before draping around the planet and being carried downtail, creating an induced magnetotail consisting of two lobes (Luhmann \& Cravens 1991). Unlike at intrinsically magnetized planets, the plane of the plasmasheet separating the two lobes is oriented perpendicular to the IMF direction, and so varies in its orientation as the IMF changes, rather than being fixed in a planet-based frame. Observations from Pioneer Venus, Venera 4, 9, and 10, and Mariner 5 were used by Saunders \& Russell (1986) to study the location of the tail boundary out to 12 Venus radii $\left(R_{V}\right)$ downtail. The more distant magnetotail region of the Venus system is not understood as well, as previous mission orbits remained relatively close to the planet.

The ionopause, or the upper boundary of the ionosphere, is located closer to Venus at an altitude of $\sim 300 \mathrm{~km}$ at the subsolar point and $\sim 1000 \mathrm{~km}$ at the dawn-dusk terminator (Phillips \& McComas 1991). The boundary between the magnetosheath and the induced magnetosphere is called the upper 
mantle boundary, and is sometimes referred to as the induced magnetospheric boundary. The boundary between the mantle and the ionosphere is called the lower mantle boundary (see Phillips \& McComas 1991, and references therein) and is also sometimes referred to as the ionopause or the ion composition boundary. This mantle region consists of plasma originating from both the solar wind and Venus, but it typically has a higher magnetic field and lower plasma density than the magnetosheath, while plasma within the lower mantle boundary is predominantly of planetary origin (Spenner et al. 1980).

Atmospheric outflow and loss lead to the presence of lowenergy $(<1 \mathrm{keV}) \mathrm{H}^{+}, \mathrm{He}^{+}$, and $\mathrm{O}^{+}$ions within the Venusian system. As with other planetary systems, energetic particles are observed within the Venusian system (see Dubinin et al. 2011, and references therein), requiring acceleration mechanisms to be present to energize or heat plasma originating from the Venusian atmosphere. However, the dominant processes accelerating the thermal Venusian plasma populations up to the suprathermal range ( $\sim 10$ 's keV) are not fully understood, especially in the distant magnetotail and magnetosheath regions.

While there have been several missions dedicated to the study of Venus and its near-space environment, such as Vega, Pioneer Venus Orbiter, and Venus Express (see Russell \& Vaisberg 1983; Futaana et al. 2017), the system has also been visited by spacecraft executing gravity assist maneuvers (GAMs) while in route to their final destination. Despite not being outfitted with instruments geared towards the Venusian system as a prime science target, these flybys have provided additional insight into the plasma populations and dynamics at Venus such as the following: observations of the Venusian foreshock by Galileo (Williams et al. 1991); detection of submicron radiation from the surface of Venus (Baines et al. 2000) and extreme ultra violet spectral investigations of the Venus dayglow (Gérard et al. 2011) by Cassini; detection of hot flow anomalies ahead of the Venusian bow shock by MESSENGER (Slavin et al. 2009); and, more recently, investigations of electric field double layers near the bow shock (Malaspina et al. 2020), subproton scale magnetic holes (Goodrich et al. 2021), and kinetic-scale turbulence in the magnetosheath (Bowen et al. 2021) by Parker Solar Probe. As such, with the successful first and future Venus GAMs of Solar Orbiter, there is a new opportunity to take measurements within this planetary system.

In this paper, we investigate the energetic particles observed within and around the Venusian system during the first Solar Orbiter Venus flyby. The following section (Sect. 2) discusses the datasets used in this study, and is followed by an overview of the Venus flyby (Sect. 3.1). A detailed investigation of various possible particle acceleration mechanisms in the Venusian tail is provided in Sect. 3.2, descriptions of the upstream suprathermal ion enhancement is given in Sect. 3.3, and an examination of galactic cosmic rays (GCRs) near Venus is discussed in sect. 3.4. Finally, a summary of these energetic particle observations and conclusions are given in Sect. 4.

\section{Datasets}

The Solar Orbiter mission (Müller et al. 2013, 2020) was launched in February 2020 to investigate the fundamental processes occurring on the Sun and in interplanetary space. To reach its final orbit, the spacecraft will execute seven Venus flybys to lower its perihelion and raise its inclination (Müller et al. 2020). Solar Orbiter consists of a wide array of in situ and remote sensing instrumentation, but only a subset of the in situ instruments were operational during the first Venus GAM, which occurred on 27 December 2020. Central to the study of the energetic particle environment encountered by the mission, Solar Orbiter is equipped with the Energetic Particle Detector (EPD) suite of sensors (Rodríguez-Pacheco et al. 2020), consisting of the Suprathermal Ion Spectrograph (SIS), the SupraThermal Electrons and Protons sensor (STEP), the Electron Proton Telescope (EPT), and the High-Energy Telescope (HET). This study uses EPD observations from only STEP and the HET C detector, as SIS was powered down for instrument safety during the GAM (see Wimmer-Schweingruber et al. 2021) and EPT and the other detectors of HET did not measure an appreciable signal. Magnetic field observations are also a key part of the in situ suite available during the Venus flyby (discussed in more detail later in this section), as these observations provide insight into possible acceleration mechanisms and contextual information of the Solar Orbiter location within the Venusian system. More information about the magnetic field observations during this Venus flyby is provided in Volwerk et al. (2021).

STEP measures both electron and the total ion populations (i.e., no species discrimination) in the suprathermal energy range and is mounted facing the sunward direction on the spacecraft. The STEP observations used in this study are from the "magnet channel" of the sensor, in which electrons are rejected through the use of a permanent magnet to allow for observations of all ions within the energy range $4-80 \mathrm{keV}$ (Rodríguez-Pacheco et al. 2020). The energies associated with the total ion flux observed by STEP are calculated under the assumption that the measured ions are all protons and have been adjusted to correspond to their primary energy (i.e., the energy before the ions lose energy in the dead layer of the STEP detector). Heavier ions can contribute to this measurement in a disproportionate manner, depending on their flux and energy spectrum (see Wimmer-Schweingruber et al. 2021, for an in-depth discussion of these instrumental effects). A constant background signal at $\sim 10 \mathrm{keV}$ in this study is a result of known instrumental background from an X-ray line at $8 \mathrm{keV}$, which is mapped to $\sim 10 \mathrm{keV}$ by this procedure (see Rodríguez-Pacheco et al. 2020).

The HET $\mathrm{C}$ detector high-gain channel count-rate product detects energetic particles (e.g., $\gtrsim 4 \mathrm{MeV}$ for protons), that are generally interpreted to be from GCRs during solar quiet periods, with a roughly isotropic response (see Freiherr von Forstner et al. 2021). For this study, the high-gain channels " $\mathrm{C} 1 \mathrm{H}$ " and " $\mathrm{C} 2 \mathrm{H}$ " count-rates are added for both HET sensors (see Freiherr von Forstner et al. 2021) for a lengthier discussion on HET and the HET $\mathrm{C}$ detector count rates). Due to the possibility of a single particle being able to trigger a count on both HET $\mathrm{C}$ photodiodes, estimates of the error associated with the count rates are not straightforward, and as such are not included in this study. Comparisons between both HET detectors and the high-gain and low-gain channels were all performed (not shown) without a significant change to the qualitative variations throughout the Venus flyby.

This study also uses magnetic field data from the Solar Orbiter fluxgate vector magnetometer (MAG, Horbury et al. 2020) at a sample cadence of 8 vectors $\mathrm{s}^{-1}$ and an electron density estimate from the Radio and Plasma Waves instrument (RPW, Maksimovic et al. 2020), since direct bulk ion measurements were not available during the first Venus GAM. The magnetic field is reported in the Venus Solar Orbital (VSO) coordinate system, in which the $x$-axis is pointed along the VenusSun line with positive being toward the Sun, the $z$-axis is parallel to the normal of the Venus orbital plane with positive being northward, and the $y$-axis completes the right-hand set (i.e., positive points toward dusk). Electron density estimates were 
R. C. Allen et al.: Energetic ions in the Venusian system: Insights from the first Solar Orbiter flyby

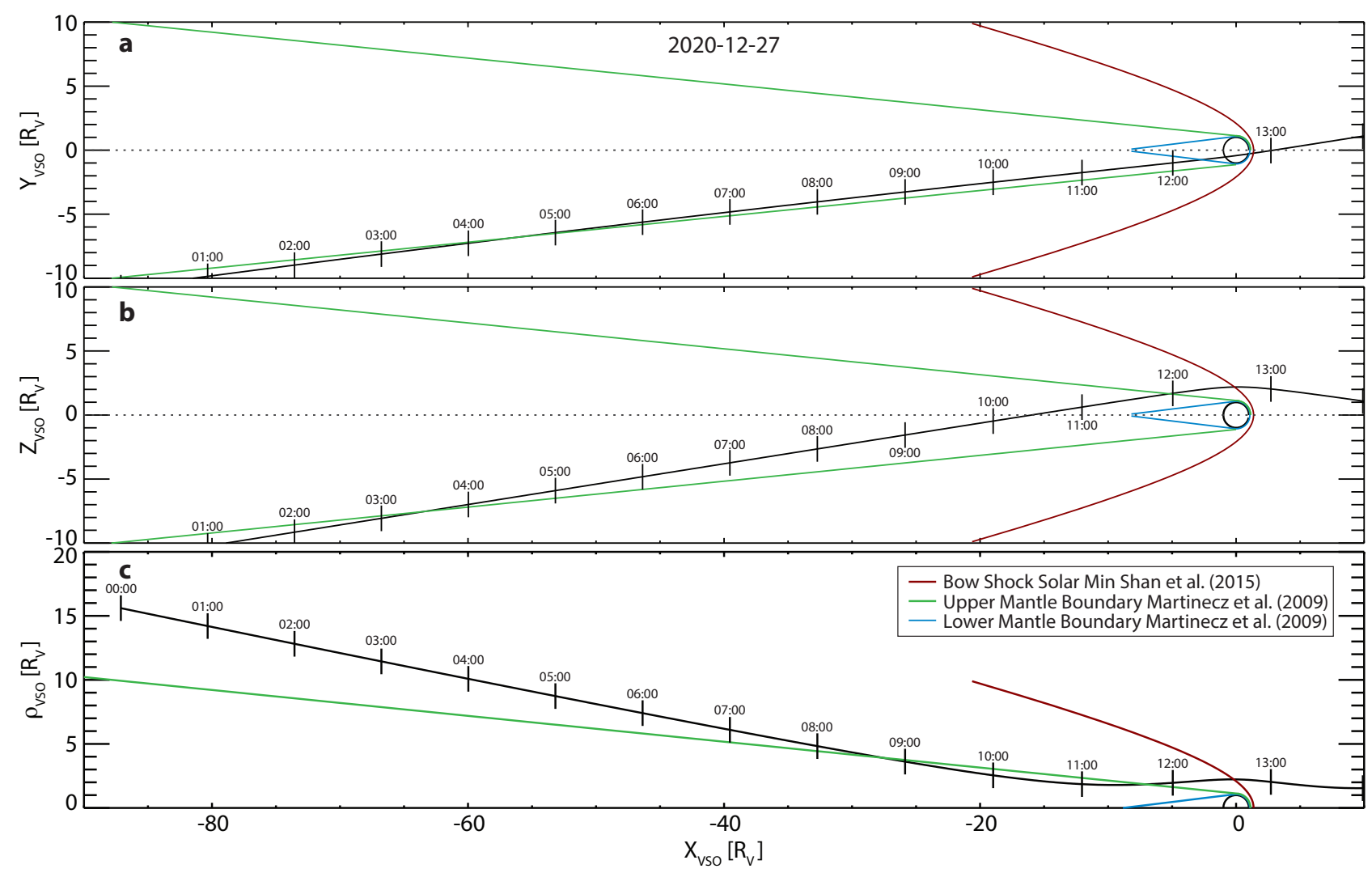

Fig. 1. Trajectory of Solar Orbiter through the Venusian system on 27 December 2020 in VSO coordinates. Empirically derived estimates of the bow shock location (Shan et al. 2015), as well as estimates of the upper mantle and lower mantle boundary locations (Martinecz et al. 2009) are provided for reference.

created by calibrating changes in the spacecraft potential (sampled at a high time resolution, up to 256 samples s$^{-1}$ ) to the plasma frequency observed in RPW measurements (sampled at a lower time resolution which varies depending on signal strength). For more information on the derivation of the electron density estimate, see Khotyaintsev et al. (2021). This study uses a $10 \mathrm{~s}$ resolution electron density estimate.

\section{Observations of the Venusian system}

\subsection{Venus flyby overview}

The trajectory of Solar Orbiter through the Venusian system on 27 December 2020 is illustrated in Fig. 1. As seen in Figs. 1a and b, Solar Orbiter approaches Venus from the dawn side, and traverses from beneath the orbital plane of Venus before crossing the orbital plane and passing over the northern pole of the planet in the VSO system. As viewed in cylindrical coordinates (with $\rho_{\mathrm{VSO}}=\sqrt{Y_{\mathrm{VSO}}^{2}+Z_{\mathrm{VSO}}^{2}}$ ) and based on empirical models of the Venus upper mantle boundary (Martinecz et al. 2009) and bow shock (Shan et al. 2015), Solar Orbiter is expected to have skimmed the upper mantle boundary from $~ 8: 00 \mathrm{UT}$ until $\sim 12: 00$ UT before crossing the bow shock on $\sim 12: 40$ UT (Fig. 1c). Just like with magnetotails in systems formed from global intrinsic magnetic fields, the Venusian magnetotail undergoes various motions due to variations in the solar wind (Zhang et al. 2009; Edberg et al. 2011; Rong et al. 2015). As such, empirically derived boundary locations in the magnetotail can only be used as an estimate of the average boundary location.
Figure 2 provides an overview of the Solar Orbiter observations during this flyby. While this study focuses on the STEP and HET $\mathrm{C}$ detector measurements, detailed overviews of the RPW and MAG observations are reported by Hadid et al. (2021) and Volwerk et al. (2021), respectively. As seen in the magnetic field observations (Figs. 2a and b), Solar Orbiter's approach to Venus is largely characterized by turbulent magnetosheath-like signatures before crossing the quasi-perpendicular bow shock at 12:40 UT (see Dimmock et al. 2021). The observed turbulence signatures and bow shock crossing are studied in more detail by Carbone et al. (2021) and Dimmock et al. (2021), respectively. The electron density estimate from RPW (Fig. 2c) shows a clear peak in density at the bow shock and a gradual rise in density with increasing radial distance from Venus downtail. The relatively high electron density throughout the flyby is consistent with Solar Orbiter largely traversing the magnetosheath. A more in-depth comparison of the estimated electron density with simulations of the solar wind-Venus interaction is presented in Stergiopoulou et al. (in prep.). Through the magnetotail region, the STEP sensor observes several bursty enhancements (Fig. 2d). The data gap from $\sim 11: 45-\sim 13: 00$ UT is due to instrument operations (see Wimmer-Schweingruber et al. 2021). After being switched off for the closest approach of Venus, STEP became operational again shortly after 13:00 UT to observe a period of decreasing flux until $\sim 13: 30$ UT when the flux reached the background of the instrument (Fig. 2d). Meanwhile, the GCR count rate is observed to be steady throughout most of the flyby with a notable decrease of $\sim 5 \%$ near the planet.

Summing the STEP observed flux across all energy channels, Fig. 3a illustrates the flux projected along the trajectory of Solar Orbiter in cylindrical coordinates. The STEP 


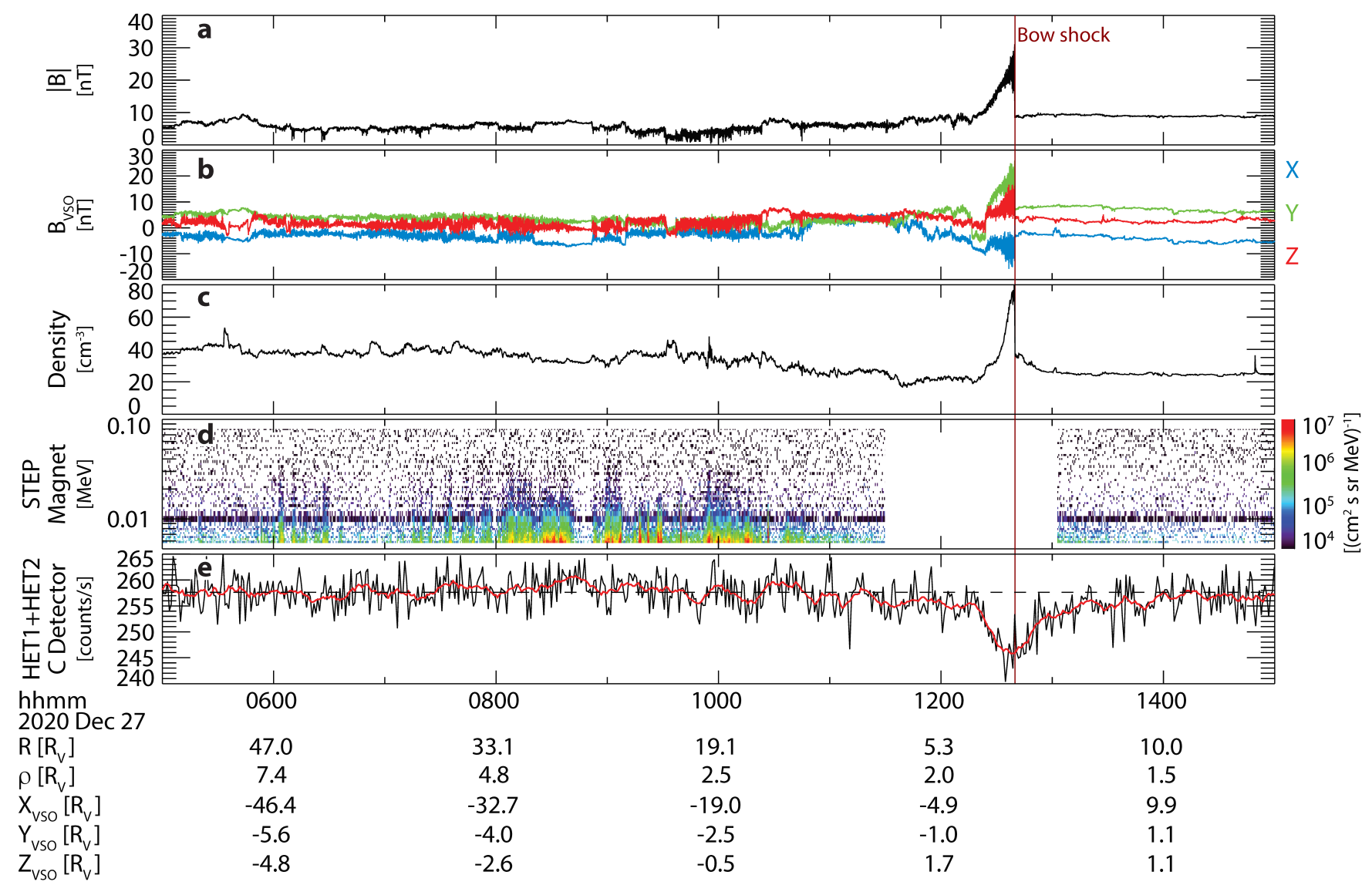

Fig. 2. Overview of observations from the Venus flyby showing the magnetic field magnitude from MAG (panel a), MAG magnetic field vector in VSO coordinates (panel b), electron density estimate derived from RPW (panel c), STEP Magnet channel total ion flux spectrogram (panel d), and HET detector $\mathrm{C}$ high-gain channel GCR count rate (panel $e$ ). The bow shock is denoted by the vertical red line. The persistently high STEP count rate around $0.01 \mathrm{MeV}$ is due to a well-understood instrumental background (Rodríguez-Pacheco et al. 2020).

enhancements are seen to correspond both to times when Solar Orbiter was near the empirical upper mantle boundary, as well as a small enhancement upstream of the bow shock. Meanwhile, the GCR count rate decrease is centered around the closest approach to Venus (Fig. 3b). These observations will be discussed further in three sections: STEP observations in the magnetotail (Sect. 3.2) and upstream regions (Sect. 3.3), and the GCR obstructions from Venus measured by HET (Sect. 3.4).

\subsection{Suprathermal ion enhancements in the Venusian magnetotail region}

Figure 4 focuses on Solar Orbiter's observations during its traversal of the Venusian magnetotail from 05:00-12:00 UT. As also shown in Figs. 2d and 3a, multiple periods of bursty particle enhancements are observed during this time, with energies reaching slightly above $30 \mathrm{keV}$. While ions reaching the 1 's $\mathrm{keV}$ energy range have been observed previously in the downtail region closer to the planet, the properties and potential acceleration mechanisms of these particles in the magnetotail region are not fully understood. Various processes have been proposed to accelerate ions up to, or explain variations in the observations of ions in, the keV range including: (1) spatial asymmetries of energetic outflow in the plasmasheet related to the solar wind convective electric field, (2) pick-up ion acceleration, (3) acceleration from strong turbulence, (4) ion cyclotron waves, (5) tail boundary layer dynamics, (6) current sheet-related acceleration, and (7) acceleration at the bow shock. To understand the dominant acceleration processes at play in the Venusian system that may have led to the STEP particle enhancements, each of these potential mechanisms are further explored in the following subsections.

\subsubsection{Asymmetries in ion outflow related to the solar wind convective electric field}

Observations from Venus Express have found that outflowing ions from Venus can escape the system through the plasma sheet of the induced magnetotail. Due to the absence of an intrinsic planetary magnetic field, the topology of the tail region of Venus is oriented along the plane containing the solar wind convective electric field $\left(E_{\mathrm{SW}}=-V_{\mathrm{SW}} \times B\right)$, unlike at magnetized planets where the plane of the plasmasheet is governed by topology of the stretched nightside magnetic field. As the ions escape through the tail region, the ions fluxes are larger within the plasma sheet and become spatially separated by energy; higher energy ions ( $\sim \mathrm{keV}$ range) are found only in the $+E_{\mathrm{SW}}$ hemisphere, while lower energy ions are found in the $-E_{\mathrm{SW}}$ hemisphere, possibly related to preferential ion escape on the side of the planet with $E_{\mathrm{SW}}$ pointed away from the planet (e.g., Phillips et al. 1987; Slavin et al. 1989; Intriligator 1989; Luhmann et al. 2006; Barabash et al. 2007; Jarvinen et al. 2013). Despite most of the observations being within the magnetosheath region of Venus, ion populations are known to be 
R. C. Allen et al.: Energetic ions in the Venusian system: Insights from the first Solar Orbiter flyby

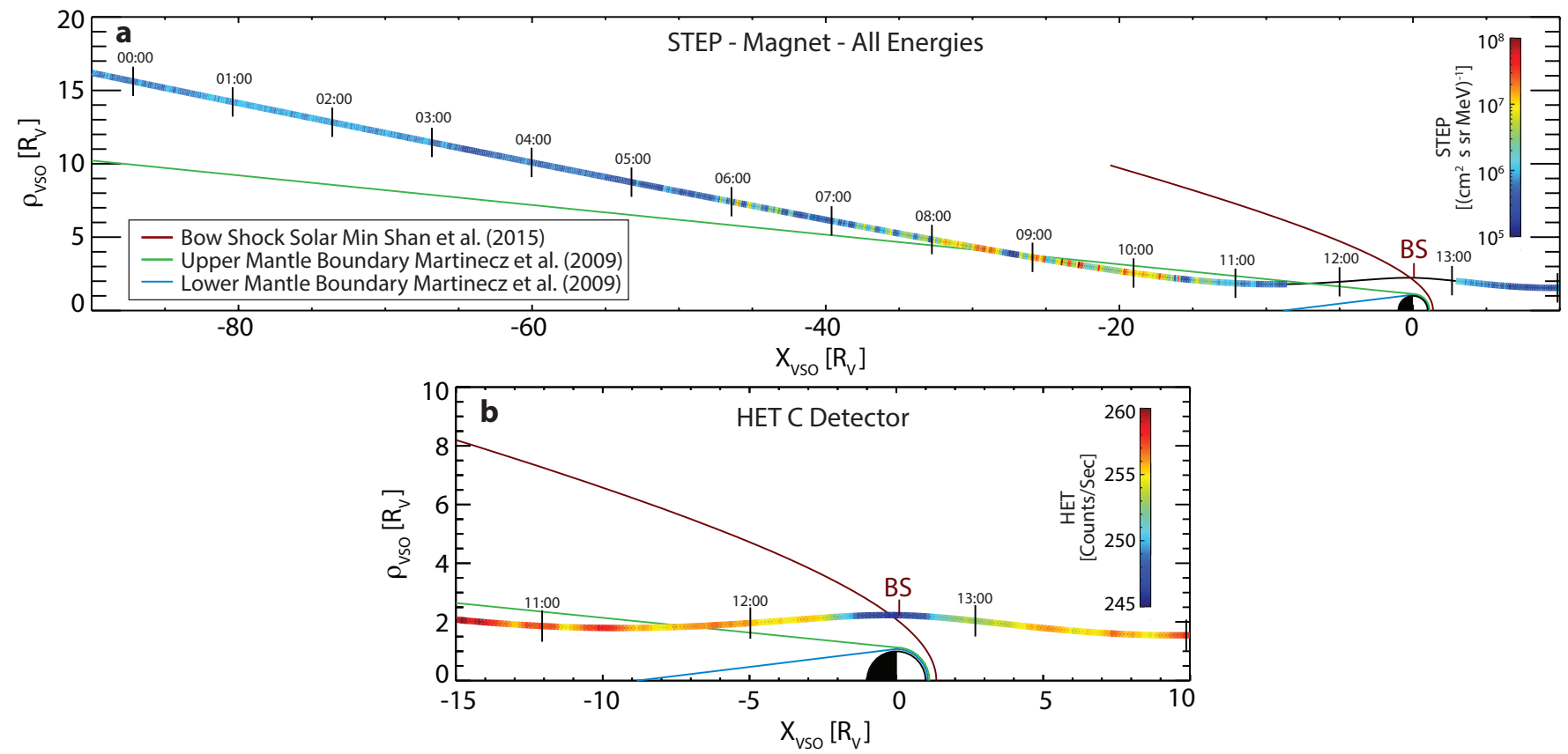

Fig. 3. Panel a: STEP magnet channel ion flux summed over all energy channels and panel $b$ : HET C detector high-gain channel count rates overplotted on the trajectory of Solar Orbiter in cylindrical VSO coordinates. Empirically derived estimates of the bow shock location (Shan et al. 2015), as well as upper mantle and lower mantle boundaries (Martinecz et al. 2009) are overplotted for reference.

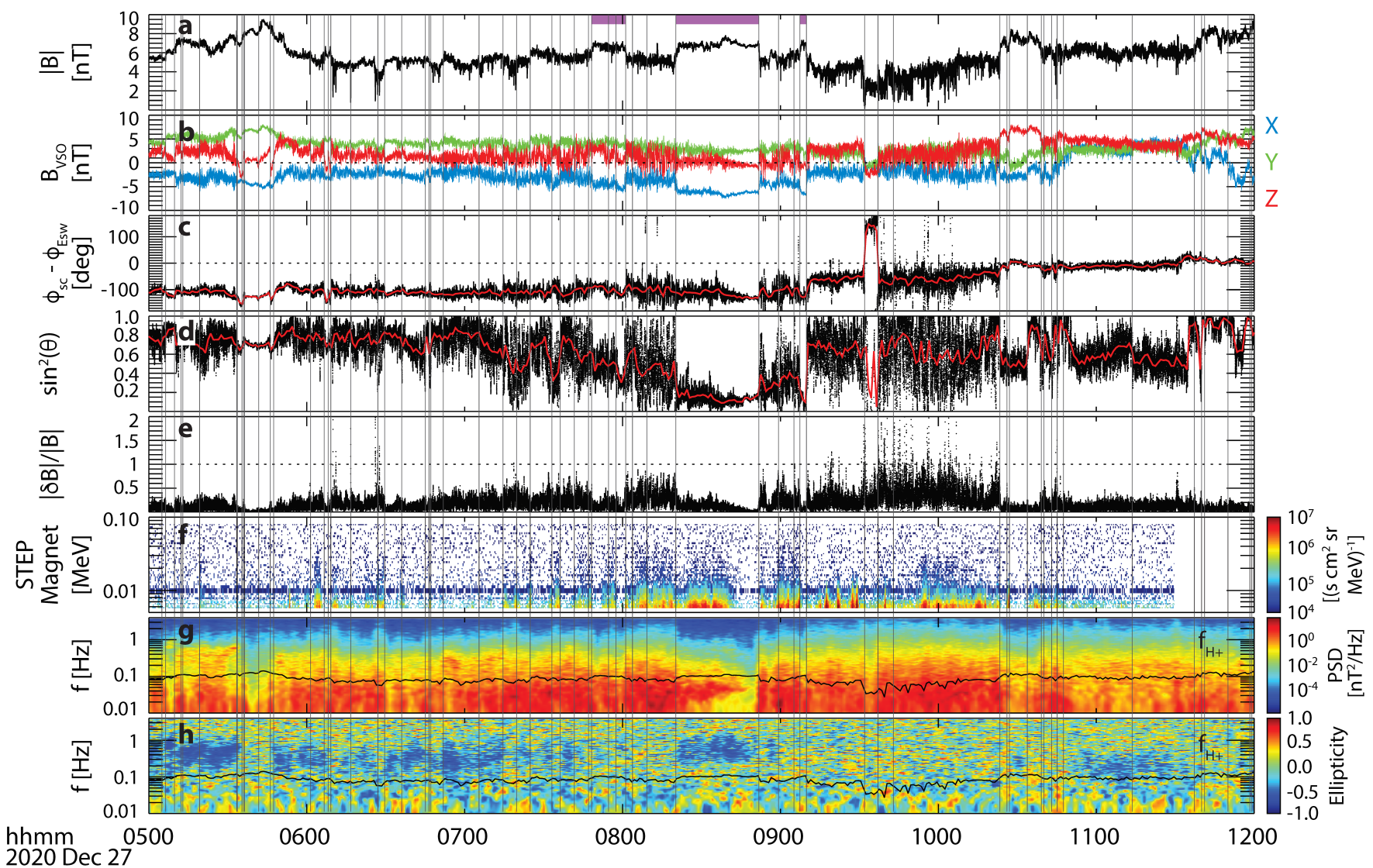

Fig. 4. Overview of the tailward pass of Venus by Solar Orbiter showing magnetic field magnitude (panel a), magnetic field vector in VSO coordinates (panel b), difference between the clock angles of the spacecraft and the solar wind convective electric field $($ panel $c)$, $\sin ^{2}(\theta)$ of the magnetic field and assumed radial bulk flow (panel d), $|\delta B| /|B|$ (panel e), STEP Magnet channel total ion flux spectrogram (panel f), power spectral density of magnetic field perturbations (panel $g$ ), and ellipticity of the magnetic field (panel $h$ ). The local proton gyrofrequency is overplotted on panels $g$ and $h$. Vertical lines denote current sheet crossings, and the purple bars in panel a mark traversals into the magnetotail (see text for criteria). 
able to both enter into, and leak out of, magnetospheres (e.g., Allen et al. 2017, 2018, 2019). In fact, Grünwaldt et al. (1995) reported possible evidence of planetary ions from Venus being detected near Earth on one occasion. As such, one potential explanation for the bursty signatures observed in the STEP magnetotail observations is that the spacecraft is skirting, or ions are leaking out of, the $+E_{\mathrm{SW}}$ hemisphere plasma sheet boundary. To test this, the clock angle of the spacecraft $\left(\phi_{\mathrm{sc}}=\right.$ $\left.\tan ^{-1}\left(Y_{\mathrm{VSO}} / Z_{\mathrm{VSO}}\right)\right)$ and solar wind electric field clock angle $\left(\phi_{\text {Esw }}=\tan ^{-1}\left(B_{Y, \mathrm{VSO}} / B_{Z, \mathrm{VSO}}\right)-90^{\circ}\right)$ is computed and the difference is shown in Fig. 4c. Times when the spacecraft trajectory is directed nearly along $+E_{\mathrm{SW}}$ (and so a difference in clock angles near $0^{\circ}$ ) correspond to times when the spacecraft would be in regions of higher energy outflow.

The difference in clock angles is large $\left(\sim-100^{\circ}\right)$ for most of the tail pass prior to $\sim 9: 00 \mathrm{UT}$, after which the spacecraft and $E_{\mathrm{SW}}$ become co-aligned (after $\sim 10: 30 \mathrm{UT}$ ). Comparing this systematic variation in $E_{\mathrm{SW}}$ to the suprathermal ion enhancements observed by STEP, there is little agreement between the relative location of Solar Orbiter with the $E_{\mathrm{SW}}$. In fact, times when Solar Orbiter becomes co-aligned with $E_{\mathrm{SW}}$ correspond to times with few to no enhancements, whereas the main STEP ion increases are seen at times when Solar Orbiter is $\sim 100^{\circ}$ away from the direction of $E_{\mathrm{SW}}$. As such, while the preferential location along the plasmasheet of suprathermal ions reported by Barabash et al. (2007) is likely still occurring within the tail region, this mechanism is likely not responsible for the STEP observations.

\subsubsection{Pick-up ion acceleration}

It has been well established that newly ionized ions can be "picked-up" by the bulk flow of plasma with an embedded magnetic field. In a fixed inertial frame, the energy gain from this "pick-up" processes can vary from zero to four times the bulk plasma speed. The maximum energy gained by this process is then

$E_{\text {max }}=2 m v_{\text {bulk }}^{2} \sin ^{2} \theta$

where $\theta$ is the angle between the magnetic field and the bulk flow. The limiting cases of pick-up ion acceleration would be when the magnetic field is perpendicular to the bulk flow (maximum energy gain) and when the magnetic field and bulk flow are parallel to one another (zero energy gain). During the Solar Orbiter Venus flyby, ion composition and bulk speed information is not available, and as such the value of $E_{\max }$ cannot be quantitatively determined. However, previous investigations at Venus have shown that the bulk flow in the magnetotail ( $>$ few $R_{V}$ ) is roughly along the negative $x$-direction in VSO coordinates (e.g., Fedorov et al. 2011; Phillips \& McComas 1991, and references therein), and as such the $\sin ^{2} \theta$ term can be computed and compared with the STEP enhancements. It should be noted that this proxy for pick-up ion acceleration is only able to investigate the possibility of local acceleration, and as such no conclusions are being made with respect to pick-up ion processes and acceleration occurring remotely from Solar Orbiter (e.g., Jarvinen et al 2020).

Comparing the value of $\sin ^{2} \theta$ (Fig. 4d) with the enhancements in STEP (Fig. 4f) does not produce good agreement. While most of the magnetic field measurements during the magnetotail pass result in $\sin ^{2} \theta \approx 0.8$, many of the STEP enhancements are observed during times of lower $\sin ^{2} \theta$, that is, the enhancements are often observed in times of more radial magnetic field orientations (relative to the rough assumption of bulk flow direction) when energy gain from the pick-up acceleration process would be small. As such, variations in the pick-up ion acceleration due to variations in $\theta$ do not explain the bursty enhancements in the suprathermal ions. It should be noted that variations in composition, thereby changing $\mathrm{m}$, or changes in the bulk speed could still change the maximum energy gain. The velocity in the downtail magnetosheath have not been observed to vary greatly (Phillips \& McComas 1991, and references therein), particularly within the relatively short timescales relevant to the suprathermal ion enhancements observed by Solar Orbiter. Therefore, variations in the bulk speed may not be playing a large role in providing the time-dependent energization from pick-up acceleration for this interval. From the observations available, it is not possible to determine if the composition is different between the times of suprathermal flux enhancement and lower enhancement. As such, it is not possible to determine if compositional variations could be playing a role in the STEP observations.

\subsubsection{Acceleration from strong turbulence}

Strong turbulence (i.e., $|\delta B| /|B| \sim 1$ ) can result in nonthermal acceleration (e.g., Ergun et al. 2020a,b). Magnetosheaths are turbulent regions, and as such parts of the magnetosheath region may have strong enough turbulence to accelerate ions into the energy range of STEP. To investigate this, $|\delta B|$ was computed by subtracting the magnetic field magnitude from the 30 -second box-car averaged background field $(|B|)$, with the resulting ratio shown in Fig. 4e. A more detailed investigation of the turbulence and intermittency across the Venus flyby is presented in Carbone et al. (2021), however this calculation reveals varying levels of turbulence throughout the tail approach.

Some of the periods with enhanced $|\delta B| /|B|$ coincide with times of enhanced suprathermal ion enhancements. Particularly the enhancement spanning from $~ 9: 30-10: 30$ UT, which occurs during the most intense magnetic fluctuations, shows good agreement with enhancements in the suprathermal ion flux. While times of large $|\delta B| /|B|$ correspond with times of elevated suprathermal ion flux, not all of the suprathermal ion enhancements are concurrent with large $|\delta B| /|B|$, suggesting that while strong turbulence is likely accelerating ions into the energy range of STEP, other processes are also contributing to the suprathermal ion abundance in the Venusian tail region. While the present analysis considers the overall turbulence intensity, further analysis could help explore the possible role of intermittency and small-scale magnetic structures (see Carbone et al. 2021).

\subsubsection{Ion cyclotron waves}

Ion cyclotron waves (ICWs) are known to occur and heat or accelerate ions in many environments (e.g., Zhang et al. 2010, 2011; Wei et al. 2011). To investigate if the enhancements in STEP are concurrent to ICWs, the magnetic field power spectral density (PSD) was generated from the Fast Fourier Transform of the magnetic field over a frequency range of 0.01 to 4 $\mathrm{Hz}$ (the Nyquist frequency of the normal magnetic field data product of Solar Orbiter). The ellipticity was computed from the ellipse transcribed by the field oscillations transverse to the background magnetic field (see Samson \& Olson 1980; Means 1972) with -1 (+1) signifying a left-hand (right-hand) circularly polarized wave and 0 representing a linearly polarized wave. The PSD and ellipticity are shown in Figs. $4 \mathrm{~g}$ and h, respectively. The local proton cyclotron frequency is overplotted in black. While there is generally broadband emission present throughout the magnetotail pass, left-hand circularly polarized waves are 
observed at times with a high degree of polarization (not shown) above the local proton cyclotron frequency. As discussed in Volwerk et al. (2021), these waves are likely ICWs that are Doppler shifted to appear above the local proton gyrofrequency in the observations. More in-depth analysis of the wave properties and discussion on their generation is given in Volwerk et al. (2021).

Investigating the timing of the ICW observations and the suprathermal ion enhancements do not generally show agreement. The main exception is the ICW observed from $\sim 08: 30$ to $\sim 08: 50 \mathrm{UT}$, which is concurrent to a broad enhancement of suprathermal ions. The other ICW observations do not well align with ion enhancements, and the other ion enhancements occur during times without ICW signatures. As such, with the exception of the $\sim 08: 30$ to $\sim 08: 50$ suprathermal ion enhancement, which may be related to heating from ICW waves, the particle enhancements are likely due to other processes. Additional flybys of Venus may be able to better constrain where (i.e., within the induced magnetotail versus the magnetosheath) ICWs may be heating the local plasma population. Beyond ICW waves, high frequency electrostatic waves could also play a role in accelerating particles (e.g., Szegö et al. 1991), and will be the focus of future investigations.

\subsubsection{Magnetotail boundary layer processes}

Previous observations from Venera 10 of the boundary layer between the magnetosheath region and the magnetotail plasma wake revealed bursty signatures in the ion temperatures (Romanov et al. 1978). Additionally, different processes such as differential streaming of electrons and ions, along with ions from the solar wind versus the ionosphere, can setup differential electric fields as well as instabilities including ion-ion instabilities and modified two-stream instabilities (see Dubinin et al. 2011, and references therein). As most of the STEP enhancements are observed in close proximity to the empirically derived upper mantle boundary (Fig. 3a), it is possible that the enhancements are related to these temperature fluctuations in the boundary layer between the magnetosheath and the upper mantle of the tail. While Solar Orbiter did not have bulk plasma velocity measurements during the Venus flyby, as was used to identify the boundary layer in Romanov et al. (1978), the magnetic field can still be used to differentiate regions. As discussed in Bertucci et al. (2011), the magnetosheath is characterized as being significantly more turbulent than the magnetotail region and the tail region tends to have a higher magnetic field strength (by a factor of $\sim 2$ ). Investigating the magnetic field fluctuations (Figs. 4b and e) as well as the magnetic field magnitude (Fig. 4a), this criterion identifies 3 likely excursions into the magnetotail region (denoted by the purple bars at the top of Fig. 4a). Boundary layers should exist at the transitions into/out of these tail regions. However, there is not sufficient information to robustly assess how Solar Orbiter is traversing this boundary, the distance from the boundary crossing, or the thickness of a boundary layer for these crossings.

Comparing the suprathermal ion enhancements (Fig. 4f) to the locations of the possible boundary layers at the transitions between the magnetotail and magnetosheath regions reveals that the boundary layers are typically associated with enhancements in the energetic particles, at least on one side of the crossing. From the observations available, it is unclear which potential processes occurring within the boundary layer may be playing a role in accelerating ions into the energy range of STEP during these crossings, however the transitions from the sheath region into the boundary layer is found to always coincide with current sheet crossings (discussed in more detail in Sect. 3.2.6). Future flybys, when more measurements may be available, such as thermal plasma observations, may provide additional insights into the acceleration mechanisms occurring in the boundary layers of the Venusian magnetotail.

\subsubsection{Current sheet-related acceleration}

Current sheets are known to be able to accelerate ions. This acceleration can result from several factors including: acceleration due to magnetic field tangential stresses (e.g., Dubinin et al. 1993, 2013), acceleration from actively reconnecting current sheets (e.g., Drake et al. 2009a,b; Vines et al. 2017), and gyroorbit effects (e.g., Speiser 1965; Chen \& Palmadesso 1986; Cowley 1978). To investigate possible correlations between the suprathermal ion enhancements and current sheet-related acceleration mechanisms, the magnetotail interval was inspected for signatures of current sheet crossings (denoted by the vertical lines in Fig. 4). These crossings were identified as an abrupt and sustained change in the magnetic field direction (i.e., not including spikes or oscillations in the data). Notably, the current sheet identified at 09:32 UT shows signs of possible magnetic reconnection (see Volwerk et al. 2021, for more details of this event).

Often, but not always, the suprathermal ions are found to be enhanced near the current sheet crossings (Fig. 4). This includes some of the relatively small, short-lived enhancements early in the approach (i.e., before 08:00 UT) when Solar Orbiter was further from the empirical upper mantle boundary. We note that, as mentioned before (Sect. 3.2.3), turbulence-generated smallscale current sheets may also contribute to particle acceleration (see, e.g., Tessein et al. 2013). The role of such structures will be investigated in a future study based on conditional statistical analysis. The fairly good correspondence between current sheet crossings and suprathermal ion enhancements suggests that current sheet-related acceleration in the Venus system is capable of accelerating ions into the energy range of STEP.

\subsection{Suprathermal ion enhancement in the upstream dayside region}

Solar Orbiter crossed the quasi-perpendicular bow shock of Venus at 12:40 UT on 27 December 2020 (see Dimmock et al. 2021, for a detailed analysis of the bow shock observations). During the bow shock crossing, STEP was turned off due to risks to the instrument related to reflected light from Venus. The STEP sensor became operable again shortly after 13:00 UT when it was $\sim 2 R_{V}$ upstream of the bow shock and observed an enhancement in lower energy $(<10 \mathrm{keV})$ ions until about 13:30 UT ( $\sim 5 R_{V}$ from the bow shock; Fig. $\left.5 \mathrm{c}\right)$. The flux of this lower energy ion enhancement decreases over time to background levels as Solar Orbiter travels further upstream of the planet. Previous observations have measured suprathermal ions upstream of the bow shock at Venus and interpreted this as being from one of two sources: (1) newly picked up ions from the neutral exosphere extending into the solar wind (e.g., Brace et al. 1988; Frank et al. 1991) or (2) foreshock particles scattered off of the Venusian bow shock (e.g., Williams et al. 1991).

During the interval upstream of Venus, Solar Orbiter observed suprathermal ions ahead of the Venusian bow shock while the IMF was directed mostly in the $+Y_{\mathrm{VSO}}$ direction (Fig. 5b). The direction of the IMF would mean that the spacecraft would not be magnetically connected to any point along the 


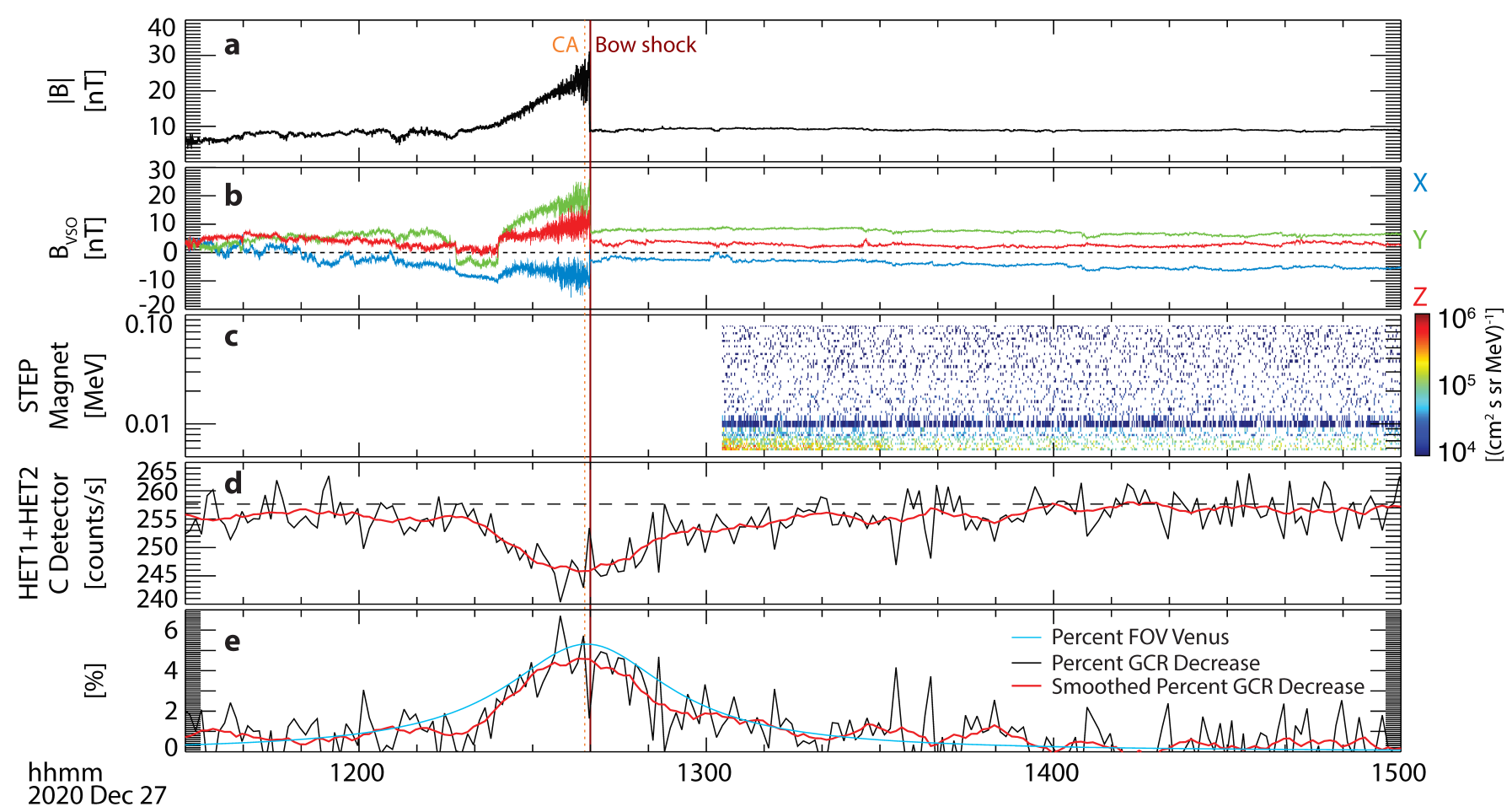

Fig. 5. Panel $a$ : magnetic field magnitude, panel $b$ : magnetic field vector in VSO coordinates, panel $c$ : STEP Magnet channel ion flux spectrogram, panel $d$ : HET C detector high-gain channel GCR count rate, and panel $e$ : percent of sky blocked by Venus (solid blue), percent decrease of GCR rate (black), and 10-min smoothed percent decrease of the GCR rate (red). The vertical solid red and dotted orange lines indicate the times of the bow shock crossing and Solar Orbiter closest approach (CA), respectively.

empirically derived bow shock from Shan et al. (2015) within reasonable uncertainty. Additionally, STEP is mounted facing the sunward direction, thereby observing ions moving toward the bow shock, rather than ion beams or reflected ions moving away from the bow shock. While foreshock ions have been observed at Venus before (e.g., Williams et al. 1991), this enhancement is not consistent with beaming or reflected ions from the Venusian bow shock, and as such must be of some other source.

Another potential explanation would be newly ionized particles, from the extended hot neutral exosphere, being picked up by the solar wind. Observations from Pioneer Venus Orbiter found ions upstream of the subsolar bow shock, which were interpreted as newly picked up ions from the exosphere (e.g., Brace et al. 1988; Moore et al. 1990; Mihalov et al. 1995; Luhmann et al. 2006). Additionally, observations from the Galileo flyby found evidence of pick-up ions in the solar wind upstream of the bow shock (Frank et al. 1991). During the time period of this enhancement, the IMF direction (assuming radial solar wind velocity) would give a value of $\sin ^{2} \theta \geq 0.9$. Assuming a nominal $400 \mathrm{~km} \mathrm{~s}^{-1}$ solar wind speed with $\sin ^{2} \theta=0.9$, the maximum expected energy for $\mathrm{O}^{+}$(a major constituent of the extended Venusian exosphere) being picked up would be $\sim 48 \mathrm{keV}$. As composition information is not available during this time period, the predominant species being observed is not known, but it is possible that these ions could be from fresh Venusian-originating pick-up ions. We cannot rule out, however, that these suprathermal ion enhancements are not of solar wind origin, as is often observed during the Solar Orbiter mission. Future flybys of the upstream region by Solar Orbiter and Parker Solar Probe may allow for a better understanding of this potential upstream population.

\subsection{Obstruction of galactic cosmic rays by Venus}

On either end of the Venus encounter, the CGR count rate from the high-gain HET $\mathrm{C}$ detector photodiodes was steady at $\sim 258$ counts $^{-1}$ (Fig. 2e). This background rate was computed using the average rate before 10:00 UT and after 14:00 UT on 27 December 2020. The GCR count rate near closest approach is shown in Fig. 5d in black, with a $10 \mathrm{~min}$ box-car averaged count rate shown in red. The steady background count rate from the pre- and post-Venus observations is shown by the dashed line. As seen by comparing the profile of the GCR decrease to the time of closest approach (vertical dotted orange line in Fig. 5), the smoothed GCR decrease is roughly symmetric about closest approach.

Figure 5e shows the percent GCR decrease of both the raw 1-min count rate (black) and 10-min smoothed GCR count rate (red). The percent of the sky that is obscured by Venus is shown by the blue curve in Fig. 5e. As the GCR count rate is taken from a data product with a roughly isotropic response, the fieldof-view of the $\mathrm{C}$ detector counters generally covers the full sky. Comparing the high-gain channels of the $\mathrm{C}$ detector count rates from HET1 and HET2 (not shown), both sensors show virtually the same percentage decrease during closest approach, further supporting the assumption that the spacecraft is not significantly affecting the look direction of the GCR count rate. Notably, the smoothed fractional GCR decrease is only slightly lower than that of the fraction of sky obscured by Venus (Fig. 5e), although the percent decrease using the 1-min count rate often reaches up to values predicted from Venus occultation.

While any difference between the fraction of sky obscured by Venus and the fractional decrease in GCR count rate is small enough to potentially be a result of instrumental uncertainty, 


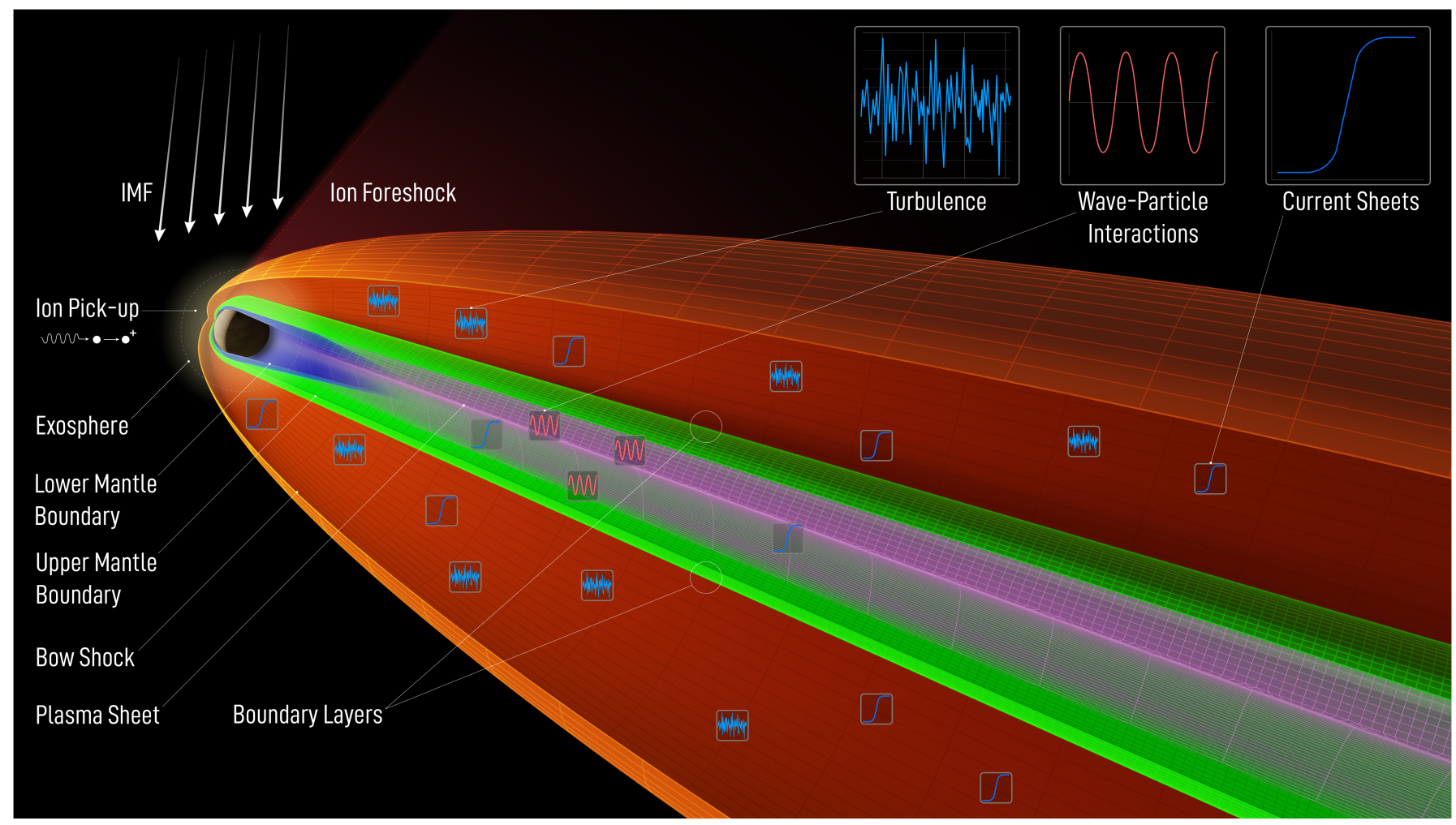

Fig. 6. Summary of acceleration processes in the Venusian system as inferred by Solar Orbiter measurements and previous observations. We note that the IMF in this diagram is $90 \sim$ from the axis of the plasmasheet (purple region), as the plasmasheet orientation is largely dictated by the IMF direction. Image credit: Ben C. Smith, JHUAPL.

it is possible that some of the discrepancy could be due to primary GCRs interacting with the atmosphere and surface of Venus, leading to the production of secondary particles (i.e., albedo particles) which are then detected by HET. Such albedo particles have been measured at Mars with the RAD instrument on Mars Science Laboratory (Appel et al. 2018) and at the Moon with the CRaTER (Schwadron et al. 2016) and LND (Wimmer-Schweingruber et al. 2020) instruments. Measured fluxes are on the order of $10 \%$ of the downward flux in the case of Mars in the energy range that can be measured by the RAD instrument (Appel et al. 2018). If these albedo particles are being produced at Venus, it would seem that their flux at $\sim 2 R_{V}$ is small. Additional Venus flybys by Solar Orbiter may help in gaining insight into the possibility of GCR albedo production at Venus.

\section{Summary and conclusions}

The first flyby of Venus by Solar Orbiter permitted a survey of the energetic particles within and around the Venusian system. In this study, one of a series of overview papers from the first Venus GAM flyby (Hadid et al. 2021; Volwerk et al. 2021; Dimmock et al. 2021), we focused on the sources and modulation of energetic particles observed by STEP and the HET C detector high-gain channel counters. The main conclusions are:

1. Bursty suprathermal ion enhancements, with energies reaching $\sim 30 \mathrm{keV}$, were observed throughout the traversal of the Venusian magnetotail and downtail magnetosheath regions at distances beyond those sampled by previous missions.

2. Asymmetries in energetic outflowing populations due to the solar wind convective electric field compared to the spacecraft location do not explain the Solar Orbiter STEP observations. As such, the suprathermal ion enhancements are likely not an effect of the spacecraft moving into/out of regions that are more aligned with the $+E_{\mathrm{SW}}$ hemisphere plasmasheet.

3. Variations in local pick-up ion acceleration from changes in the magnetic field direction do not explain the suprathermal ion enhancements observed during the Venus flyby, and so significant energy gain via the local pick-up process is not likely contributing to the observations.

4. Strong turbulence (i.e., $|\delta B| /|B| \sim 1$ ) in the magnetosheath is likely accelerating ions into the energy range of STEP.

5. Ion cyclotron waves in the magnetosheath are not seen to be likely contributors of heating ions into the range of STEP, however, there is an instance of an ICW wave within the magnetotail region of Venus that may be associated with a suprathermal ion enhancement. This may suggest ICWs play a more important role in the mantle than in the sheath region.

6. Magnetotail boundary layer dynamics may be associated with some of the suprathermal ion enhancements. The boundaries between the magnetotail and magnetosheath regions of the Venus system are often associated with suprathermal flux enhancements, and also found to coincide with current sheet crossings.

7. Current sheet crossings are observed to often have an associated increase in suprathermal ion flux. As such, current sheet processes, such as reconnection and gyroradius effects, are likely accelerating ions in the Venusian system. This is observed both in the magnetosheath away from the upper mantle boundary, as well as near and at the boundary between the sheath and upper mantle.

8. Suprathermal ion enhancements are observed as far as $\sim 5 R_{V}$ upstream of the quasi-perpendicular bow shock crossing. Due to the orientation of the IMF (mostly in the $+Y_{\mathrm{VSO}}$ direction), it is unlikely that Solar Orbiter would have been 
magnetically connected to the bow shock. Additionally, as STEP was observing particles traveling toward Venus, rather than beaming away, it is unlikely that this population was related to a foreshock. Previous studies (e.g., Brace et al. 1988; Frank et al. 1991; Jarvinen et al. 2020) have suggested that exospheric neutrals may be photoionized upstream of the bow shock before being picked up into the solar wind flow, and so may be contributing to the suprathermal enhancement measured by STEP. However, without composition or lower energy measurements we are unable to determine if this possibility fully explains the observations.

9. The GCR count rate decreases near the planet, associated with blockage of energetic particles by Venus. While GCR albedo particles have been observed at both Mars and the Moon, there does not seem to be a significant effect of these ions on the HET GCR count rate near Venus.

The main processes leading to particle acceleration in the Venusian system inferred by observations in this study, as well as other previous works (e.g., Phillips \& McComas 1991, and references therein), are illustrated in Fig. 6. These different processes, list them, have been observed to exist throughout the system, and are all likely to be contributing to the presence of suprathermal particles throughout the Venusian system. The prevalence, effectiveness, and interplay between these different mechanisms warrant future investigations to better characterize the dominant processes for, and impacts of, energetic populations within the system. Future Venus flybys by Solar Orbiter will allow for continued investigation into the suprathermal and energetic populations within the system. Different trajectories, along with potentially additional datasets during the future Venus GAMs, will not only allow insight into how consistent these observations are from one encounter to the next, but also allow for deeper investigations into the various processes accelerating ions within the Venusian system.

Acknowledgements. Solar Orbiter is a mission of international cooperation between ESA and NASA, operated by ESA. We thank the many individuals at ESA and within the Energetic Particle Detector team for their support in its development. Work on this study was funded by NASA contract NNN06AA01C, and we thank NASA headquarters and the NASA/GSFC Solar Orbiter project office for their continuing support. The UAH team acknowledges the financial support by the Spanish Ministerio de Ciencia, Innovación y Universidades FEDER/MCIU/AEI Projects ESP2017-88436-R and PID2019104863RB-I00/AEI/10.13039/501100011033. The CAU Kiel team thanks the German Federal Ministry for Economic Affairs and Energy and the German Space Agency (Deutsches Zentrum für Luft- und Raumfahrt, e.V., (DLR)) for their unwavering support under grant numbers 50OT0901, 50OT1202, 50OT1702 and 50OT2002; and ESA for supporting the build of SIS under contract number SOL.ASTR.CON.00004, and the University of Kiel and the Land Schleswig-Holstein for their support. The Solar Orbiter magnetometer was funded by the UK Space Agency (grant ST/T001062/1). Additional support was provided from the Science Technology Facilities Council (STFC) grant ST/S000361/1. The RPW instrument has been designed and funded by CNES, CNRS, the Paris Observatory, The Swedish National Space Agency, ESA-PRODEX and all the participating institutes. APD received financial support from the Swedish National Space Agency (Grant \#2020-00111).

\section{References}

Allen, R. C., Livi, S. A., Vines, S. K., et al. 2017, J. Geophys. Res. Space Phys., 122,8353

Allen, R. C., Mitchell, D. G., Paranicas, C. P., et al. 2018, J. Geophys. Res. Space Phys., 123, 4712

Allen, R. C., Paranicas, C. P., Bagenal, F., et al. 2019, Geophys. Res. Lett., 46 11709

Appel, J. K., Köhler, J., Guo, J., et al. 2018, Earth Space Sci., 5, 2

Baines, K. H., Bellucci, G., Bibring, J.-P., et al. 2000, Icarus, 148, 307

Barabash, S., Fedorov, A., Sauvaud, J. J., et al. 2007, Nature, 450, 650
Bertucci, C., Mazelle, C., Slavin, J. A., Russell, C. T., \& Acuña, M. H. 2003, Geophys. Res. Lett., 30, 1876

Bertucci, C., Duru, F., Edberg, N., et al. 2011, Space Sci. Rev., 162, 113

Bowen, T. A., Bale, S. D., Bandyopadhyay, R., et al. 2021, Geophys. Res. Lett., 48, e2020GL090783

Brace, L. H., Theis, R. F., Curtis, S. A., \& Parker, L. W. 1988, J. Geophys. Res., 93, A11

Carbone, F., Sorriso-Valvo, L., Khotyaintsev, Yu. V., et al. 2021, A\&A, 656, A16 (SO Cruise Phase SI)

Chen, J., \& Palmadesso, P. J. 1986, J. Geophys. Res. Space Phys., 91, 1499

Cowley, S. W. H. 1978, Planet. Space Sci., 26, 1037

Dimmock, A. P., Khotyaintsev, Yu. V., Lalti, A., et al. 2021, A\&A, submitted (SO Cruise Phase SI)

Drake, J. F., Cassak, P. A., Shay, M. A., Swisdak, M., \& Quataert, E. 2009a, ApJ, 700, L16

Drake, J. F., Swisdak, M., Phan, T. D., et al. 2009b, J. Geophys. Res., 114, A05111

Dubinin, E., Lundin, R., Norberg, O., \& Pissarenko, N. 1993, J. Geophys. Res. Space Phys., 98, 3991

Dubinin, E., Fraenz, M., Fedorov, A., et al. 2011, Space Sci. Rev., 162, 173

Dubinin, E., Fraenz, M., Zhang, T. L., et al. 2013, J. Geophys. Res. Space Phys., 118,7624

Edberg, N. J. T., Nilsson, H., Futaana, Y., et al. 2011, J. Geophys. Res., 116, A09308

Ergun, R. E., Ahmadi, N., Kromyda, L., et al. 2020a, ApJ, 898, 153

Ergun, R. E., Ahmadi, N., Kromyda, L., et al. 2020b, ApJ, 898, 154

Fedorov, A., Barabash, S., Sauvaud, J.-A., et al. 2011, J. Geophys. Res., 116, A07220

Frank, L. A., Paterson, W. R., Ackerson, K. L., Coroniti, F. V., \& Vasyliunas, V. M. 1991, Science, 253, 5027

Freiherr von Forstner, J. L., Dumbović, M., Möstl, C., et al. 2021, A\&A, 656, A1 (SO Cruise Phase SI)

Futaana, Y., Wieser, G. S., Barabash, S., \& Luhmann, J. G. 2017, Space Sci. Rev., 212, 1453

Gérard, J.-C., Hubert, B., Gustin, J., et al. 2011, Icarus, 211, 70

Goodrich, K. A., Bonnell, J. W., Curry, S., et al. 2021, Geophys. Res. Lett., 48, e90329

Gröller, H., Shematovich, V. I., Lichtenegger, H. I. M., et al. 2010, J. Geophys. Res., 115, E12017

Grünwaldt, H., Neugebauer, M., Hilchenbach, M., et al. 1995, Geophys. Res. Lett., 24, 1163

Hadid, L. Z., Edberg, N. J. T., Chust, T., et al. 2021, A\&A, 656, A18 (SO Cruise Phase SI)

Horbury, T. S., O’Brien, H., Carrasco Blazquez, I., et al. 2020, A\&A, 642, A9

Intriligator, D. S. 1989, Geophys. Res. Lett., 16, 167

Jarvinen, R., Kallio, E., \& Dyadechkin, S. 2013, J. Geophys. Res. Space Phys., 118,4551

Jarvinen, R., Alho, M., Kallio, E., \& Pulkkinen, T. I. 2020, Geophys. Res. Lett., 47, e2020GL087462

Khotyaintsev, Yu. V., Graham, D. B., Vaivads, A., et al. 2021, A\&A, 656, A19 (SO Cruise Phase SI)

Lu, Q. M., Shan, L. C., Zhang, T. L., et al. 2013, ApJ, 773, L24

Luhmann, J. G. 1986, Space Sci. Rev., 44, 241

Luhmann, J. G., \& Cravens, T. E. 1991, Space Sci. Rev., 55, 201

Luhmann, J. G., Kasprzak, W. T., \& Russell, C. T. 2006, J. Geophys. Res., 112, E04S10

Maksimovic, M., Bale, S. D., Chust, T., et al. 2020, A\&A, 643, A12

Malaspina, D. M., Goodrich, K., Livi, R., et al. 2020, Geophys. Res. Lett., 47, e2020GL090115

Martinecz, C., Boesswetter, A., Fränz, M., et al. 2009, J. Geophys. Res., 114, E00B30

Means, J. D. 1972, J. Geophys. Res., 77, 5551

Mihalov, J. D., Russell, C. T., Kasprzak, W. T., \& Knudsen, W. C. 1995, J. Geophys. Res., 100, 19579

Moore, K. R., McComas, D. J., Russell, C. T., \& Mihalov, J. D. 1990, J. Geophys. Res., 95, 12005

Müller, D., Marsden, R. G., St Cyr, O. C., Gilbert, H. R., \& The Solar Orbiter Team 2013, Sol. Phys., 285, 25

Müller, D., St. Cyr, O. C., Zouganelis, I., Gilber, H. E., \& Marsden, R. 2020, A\&A, 642, A1

Phillips, J. L., \& McComas, D. J. 1991, Space Sci Rev., 55, 1

Phillips, J. L., Luhmann, J. G., Russell, C. T., \& Moore, K. R. 1987, J. Geophys. Res., 92, 9920

Rodríguez-Pacheco, J., Wimmer-Schweingruber, R. F., Mason, G. M., et al. 2020, A\&A, 642, A7

Romanov, S. A., Smirnov, V., \& Vaisberg, O. 1978, Kosmich. Issled., 16, 746

Rong, Z. J., Barabash, S., Stenberg, G., et al. 2015, J. Geophys. Res. Space Phys., 120,5593 
R. C. Allen et al.: Energetic ions in the Venusian system: Insights from the first Solar Orbiter flyby

Russell, C. T., Elphic, R. C., \& Slaven, J. A. 1979, Nature, 282, 815

Russell, C. T., \& Vaisberg, O. 1983, in Venus, eds. D. M. Hunten, L. Colin, T.

M. Donahue, \& V. I. Moroz (Tucson, Arizona: Univ. of Arizona Press), 873

Samson, J. C., \& Olson, J. V. 1980, Geophys. J. R. Astron. Soc., 61, 115

Saunders, M. A., \& Russell, C. T. 1986, J. Geophys. Res., 91, 5589

Schwadron, N. A., Wilson, J. K., Looper, M. D., et al. 2016, Icarus, 273, 25

Shan, L., Lu, Q., Mazelle, C., et al. 2015, Planet. Space Sci., 109-110, 32

Slavin, J. A., Elphic, R. C., Russell, C. T., Wolfe, J. H., \& Intriligator, D. S. 1979 Geophys. Res. Lett., 6, 901

Slavin, J. A., Intriligator, D. S., \& Smith, E. J. 1989, J. Geophys. Res., 94, 2383

Slavin, J. A., Acuña, M. H., Anderson, B. J., et al. 2009, Geophys. Res. Lett., 36, L09106

Speiser, T. W. 1965, J. Geophys. Res., 70, 4219

Spenner, K., Knudsen, W. C., Miller, K. L., et al. 1980, J. Geophys. Res., 85, 7655

Szegö, K., Shapiro, V. S., Schevchenko, V. I., et al. 1991, Geophys. Res. Lett., 18,2305

Tessein, J. J., Mattaeus, W. H., Wan, M., et al. 2013, ApJ, 776, L8

Vines, S. K., Fuselier, S. A., Trattner, K. J., et al. 2017, J. Geophys. Res. Space Phys., 122, 10247

Volwerk, M., Zhang, T. L., Delva, M., et al. 2008, J. Geophys. Res., 113, E00B16

Volwerk, M., Horbury, T. S., Woodham, L. D., et al. 2021, A\&A, 656, A11 (SO Cruise Phase SI)

Vörös, Z., Zhang, T. L., Volwerk, M. P., et al. 2008, Geophys. Res. Lett., 35, L11102

Wei, H. Y., Russell, C. T., Zhang, T. L., \& Balanco-Cano, X. 2011, Planet. Space Sci., 59, 1039

Williams, D. J., McEntire, R. W., Krimigis, S. M., et al. 1991, Science, 253, 1525

Wimmer-Schweingruber, R. F., Yu, J., Böttcher, S. I., et al. 2020, Space Sci. Rev. 216, 104

Wimmer-Schweingruber, R. F., Janitzek, N. P., Pacheco, D., et al. 2021, A\&A 656, A22 (SO Cruise Phase SI)

Zhang, T. L., Delva, M., Baumjohann, W., et al. 2008, J. Geophys. Res., 113, E00B20

Zhang, T. L., Du, J., \& Ma, Y. J. 2009, Geophys. Res. Lett., 36, L20203

Zhang, J.-C., Kistler, L. M., \& Mouikis, C. G. 2010, J. Geophys. Res., 115, A06212

Zhang, J.-C., Kistler, L. M., \& Mouikis, C. G. 2011, J. Geophys. Res., 116, A11201

1 Johns Hopkins Applied Physics Lab, Laurel, MD 20723, USA e-mail: Robert.Allen@jhuapl.edu

2 Space Research Group, Universidad de Alcalá, Alcalá de Henares, Madrid, Spain
3 Institut für Experimentelle und Angewande Physik, ChristianAlbrechts-Universität zu Kiel, 24118 Kiel, Germany

${ }^{4}$ Swedish Institute of Space Physics (IRF), Uppsala, Sweden

5 Imperial College, London, UK

${ }^{6}$ LESIA, Observatoire de Paris, Université PSL, CNRS, Sorbonne Université, Univ. Paris Diderot, Sorbonne Paris Cité, 5 place Jules Janssen, 92195 Meudon, France

7 LPP, CNRS, Ecole Polytechnique, Sorbonne Université, Observatoire de Paris, Université Paris-Saclay, Palaiseau, Paris, France

8 Space Research Institute, Austrian Academy of Sciences, Graz, Austria

9 CNR/ISTP - Istituto per la Scienza e Tecnologia dei Plasmi, Via Amendola 122/D, 70126 Bari, Italy

${ }^{10}$ Space Sciences Laboratory, University of California, Berkeley, CA, USA

11 Physics Department, University of California, Berkeley, CA, USA

12 Now at DSI Datensicherheit GmbH, Rodendamm 34, 28816 Stuhr, Germany

13 LPC2E, CNRS, 3A avenue de la Recherche Scientifique, Orléans, France

14 Université d'Orléans, Orléans, France

15 Now at Deutsches Elektronen-Synchrotron (DESY), Platanenallee 6, 15738 Zeuthen, Germany

16 CNES, Toulouse, France

17 Now at German Aerospace Center (DLR), Department of Extrasolar Planets and Atmospheres, Berlin, Germany

18 Technische Universität Dresden, Dresden, Germany

19 Now at Max-Planck-Institute for Solar System Research, Göttingen, Germany

${ }^{20}$ Institute of Atmospheric Physics of the Czech Academy of Sciences, Prague, Czech Republic

21 Astronomical Institute of the Czech Academy of Sciences, Prague, Czech Republic

22 Now at Univ. Colorado/LASP, Boulder, CO, USA

${ }^{23}$ Department of Space and Plasma Physics, School of Electrical Engineering and Computer Science, Royal Institute of Technology, Stockholm, Sweden

24 Research Institute for Mathematics, Astrophysics and Particle Physics Radboud University, Nijmegen, The Netherlands 